\title{
Child eating behaviours are associated with weight status at two years of age
}

\author{
E. K. McCarthy ${ }^{1}$, D. M. Murray ${ }^{2,3}$, L. C. Kenny ${ }^{3}$, J. O’B. Hourihane ${ }^{2}$ and M. Kiely ${ }^{1,3}$ \\ ${ }^{1}$ Maternal and Infant Nutrition Research Group, School of Food and Nutritional Sciences, University College Cork, \\ ${ }^{2}$ Department of Paediatrics and Child Health, University College Cork and ${ }^{3}$ The Irish Centre for Fetal and Neonatal \\ Translational Research, Department of Obstetrics and Gynaecology, University College Cork
}

The Children's Eating Behaviour Questionnaire (CEBQ) has been validated as a measure of eating behaviours in children ${ }^{(1)}$. Associations between eating behaviours and weight status have been identified in older children ${ }^{(2,3)}$, however limited research has been carried out in young children. The aim of this analysis was to explore the relationship between eating behaviours and weight status in two year old children.

The CEBQ was completed by the parents of participants of the Cork BASELINE Birth Cohort Study at each child's 24-month assessment(median [IQR] age of $2 \cdot 1$ [2·1, 2 2$]$ years). The CEBQ encompasses eight eating behaviour subscales (four food approach, four food avoidant) and each subscale has three-five questions. Questions are assessed based on a five-point scale and scored appropriately. Anthropometric measures were also taken and participants were categorised into three weight categories (underweight, normal weight, overweight/obese) based on the International Obesity Task Force BMI cut-offs for children ${ }^{(4)}$. Only participants with complete anthropometric and eating behaviour data were included in the analysis, with 1189 participants $(50 \%$ male) included.

There were no significant differences in subscale scores between males and females, except that females scored higher in "slowness in eating" while males scored higher in "food fussiness" $(P<0 \cdot 05)$. There were significant differences in subscale scores between the three weight categories, see table. Participants in the overweight/obese category scored highest in food approach behaviours ("enjoyment of food", "food responsiveness"), while those in the underweight category scored highest in food avoidant behaviours ("satiety responsiveness", "slowness in eating", "food fussiness").

\begin{tabular}{|c|c|c|c|c|}
\hline & Underweight 77 & Normal weight 947 & Overweight/Obese 165 & P-value \\
\hline & Median [25th, 75th] & Median [25th, 75th] & Median [25th, 75th] & \\
\hline \multicolumn{5}{|l|}{ Food Approach Subscales } \\
\hline Enjoyment of Food & $3 \cdot 8[3 \cdot 0,4 \cdot 1]^{\mathrm{a}}$ & $4 \cdot 0[3 \cdot 5,4 \cdot 5]^{\mathrm{b}}$ & $4 \cdot 3[3 \cdot 8,4 \cdot 8]^{\mathrm{c}}$ & $0 \cdot 000$ \\
\hline Emotional Overeating & $1 \cdot 5[1 \cdot 0,1 \cdot 8]$ & $1 \cdot 5[1 \cdot 0,1 \cdot 8]$ & $1 \cdot 5[1 \cdot 0,2 \cdot 0]$ & $0 \cdot 153$ \\
\hline Desire to Drink & $2 \cdot 7[1 \cdot 8,3 \cdot 5]$ & $2 \cdot 7[2 \cdot 0,3 \cdot 3]$ & $2 \cdot 7[2 \cdot 3,3 \cdot 7]$ & $0 \cdot 128$ \\
\hline Food Responsiveness & $1 \cdot 0[1 \cdot 0,2 \cdot 0]^{\mathrm{a}}$ & $1 \cdot 1[1 \cdot 0,2 \cdot 0]^{\mathrm{b}}$ & $2 \cdot 0[1 \cdot 0,3 \cdot 0]^{\mathrm{c}}$ & $0 \cdot 000$ \\
\hline Grouped Food Approach Score & $2 \cdot 3[2 \cdot 0,2 \cdot 6]^{\mathrm{a}}$ & $2 \cdot 5[2 \cdot 1,2 \cdot 8]^{\mathrm{b}}$ & $2 \cdot 7[2 \cdot 4,3 \cdot 0]^{\mathrm{c}}$ & $0 \cdot 000$ \\
\hline \multicolumn{5}{|l|}{ Food Avoidant Subscales } \\
\hline Satiety Responsiveness & $3 \cdot 0[2 \cdot 7,3 \cdot 7]^{\mathrm{a}}$ & $2 \cdot 7[2 \cdot 3,3 \cdot 0]^{\mathrm{b}}$ & $2 \cdot 3[2 \cdot 0,3 \cdot 0]^{\mathrm{c}}$ & $0 \cdot 000$ \\
\hline Slowness in Eating & $3 \cdot 0[2 \cdot 7,3 \cdot 7]^{\mathrm{a}}$ & $2 \cdot 7[2 \cdot 3,3 \cdot 3]^{\mathrm{b}}$ & $2 \cdot 6[2 \cdot 3,3 \cdot 0]^{\mathrm{c}}$ & $0 \cdot 000$ \\
\hline Food Fussiness & $2 \cdot 7[2 \cdot 2,3 \cdot 8]^{\mathrm{a}}$ & $2 \cdot 5[2 \cdot 0,3 \cdot 0]^{\mathrm{b}}$ & $2 \cdot 3[1 \cdot 8,2 \cdot 9]^{\mathrm{c}}$ & $0 \cdot 001$ \\
\hline Emotional Undereating & $2 \cdot 8[2 \cdot 0,3 \cdot 8]$ & $3 \cdot 0[2 \cdot 3,3 \cdot 5]$ & $3 \cdot 0[2 \cdot 3,3 \cdot 5]$ & $0 \cdot 812$ \\
\hline Grouped Food Avoidant Score & $3 \cdot 0[2 \cdot 7,3 \cdot 7]^{\mathrm{a}}$ & $2 \cdot 8[2 \cdot 4,3 \cdot 1]^{\mathrm{b}}$ & $2 \cdot 6[2 \cdot 3,2 \cdot 9]^{\mathrm{c}}$ & $0 \cdot 000$ \\
\hline
\end{tabular}

${ }^{\mathrm{a}, \mathrm{b}, \mathrm{c}}$ Different superscript letters denote statistically differences between groups $(P<0 \cdot 05)$.

Child eating behaviours as assessed by the CEBQ were associated with weight status at two years of age. Food approach eating behaviours including "enjoyment of food" and "food responsiveness" appear to be associated with overweight and obesity. The identification of relationships between eating behaviours and food and nutrient intake patterns may provide means of an intervention strategy against later overweight and obesity.

Research supported by National Children's Research Centre.

1. Wardle J, Guthrie CA, Sanderson S, et al. (2001) J Child Psychol Psychiat 42(7), 963-70.

2. Viana V, Sinde S, Saxton JC (2008) Br J Nutr 100(2), 445-50.

3. Webber L, Hill C, Saxton J, et al. (2009) Int J Obes 33(1), 21-8.

4. Cole TJ, Bellizzi MC, Flegal KM, et al. (2000) BMJ 320(7244), 1240-3. 